\title{
VICISITUDES DE UN SENCILLO INVENTOR
}

Francisco García López.

Hospital Universitario de Elche. Elche. Alicante. España.
Resumen.- Procedimiento mecanizado para el tratamiento de la Incontinencia Urinaria de Esfuerzo en la mujer. Fijación retropúbica de la vagina con una grapa disparada con grapadora diseñada con la forma apropiada, que utiliza la sínfisis del pubis como lugar de anclaje.

Se pretende demostrar la razón fisiopatológica para devolver la continencia, por medio del anclaje de la uretra. En nuestras condiciones elementales de investigación y desarrollo no se pudo llegar a diseñar el procedimiento idoneo, aunque se presentó la idea mecanizada a Empresas de ámbito internacional.

Se relata resumidamente el desarrollo del instrumento hasta conseguir hacerlo operativo, filmándose una inter- vención que se presentó al Congreso Hispanoamericano que se realizó en Madrid en 1992.

El instrumento se patentó en la Oficina Española de Patentes y Marcas en julio de 1992 con categoría de Patente de Invención, al no existir antecedentes de procedimientos mecanizados para la fijación de la uretra a través de la vagina.

Palabras clave: Incontinencia Urinaria de Esfuerzo en la mujer. Grapado vaginal retropúbico. Historia de la Urología.

Summary.- Mechanized procedure for the treatment of female stress urinary incontinence. Retropubic fixation of the vagina with a stapler specifically designed, using the pubis' symphysis as the site for anchoring. We intend to demonstrate the physiopathologic rationale to achieve continence by anchoring the urethra. In our elementary conditions for research and development we could not design the suitable procedure, although the idea was presented to international companies.

We summarize the development of the instrument until it was operative, filming one operation which was presented to the Hispanic-American Congress in Madrid 1992.

The instrument was patented in the Spanish Office of Patents and Trademarks in July 1992 with the category of invention patent because there were no previous mechanized procedures for vaginal fixation.

Keywords: Female stress urinary incontinence. Surgical treatment. Retropubic vaginal fixation. History of Urology. 


\section{INTRODUCCIÓN}

Como suele ser normal, los fracasos son más inquietantes que los éxitos; al menos esa fué mi experiencia después de una intervención para incontinencia de orina en una paciente con connotaciones familiares. La inquietud se estableció en mi mente y fué la ocasión para idear un artilugio que tratara mecanizadamente la Incontinencia Urinaria al Esfuerzo en la mujer. Dicha idea acabó presentándose en la Oficina de Patentes el día 15 de julio de 1992 (1) y seguramente fue el primer utensilio mecanizado que se patentó con esta intención en la Historia de la Medicina, pues por entonces no se encontró nada en esa línea en toda la extensa bibliografía revisada, ni tampoco en la búsqueda del Estado de la Técnica que realiza la Oficina de Patentes. Fue por ello que se le dió la consideración como Patente de Invención.

A principio de los 80 se implanta la técnica quirúrgica que el Dr. Slomo Raz (2) prodigaba en todos los foros de esta materia. Me pareció una técnica atractiva que permitía en mi formación profesional, acercarme a una patología que hasta ese momento no era para mi lo suficiente conocida. No obstante, cuando recorrí la Historia de la cirugía reparadora de la incontinencia de esfuerzo, justo es reconocer que la intervención que prodigara el Dr. Slomo Raz, pertenecía originalmente a A.J. Pereyra publicada en 1959 (3), que luego modifican otros cirujanos.

Realicé un determinado número de intervenciones con la variante de $S$. Raz y fue con la intervención de la paciente $B$. G. que en el postoperatorio

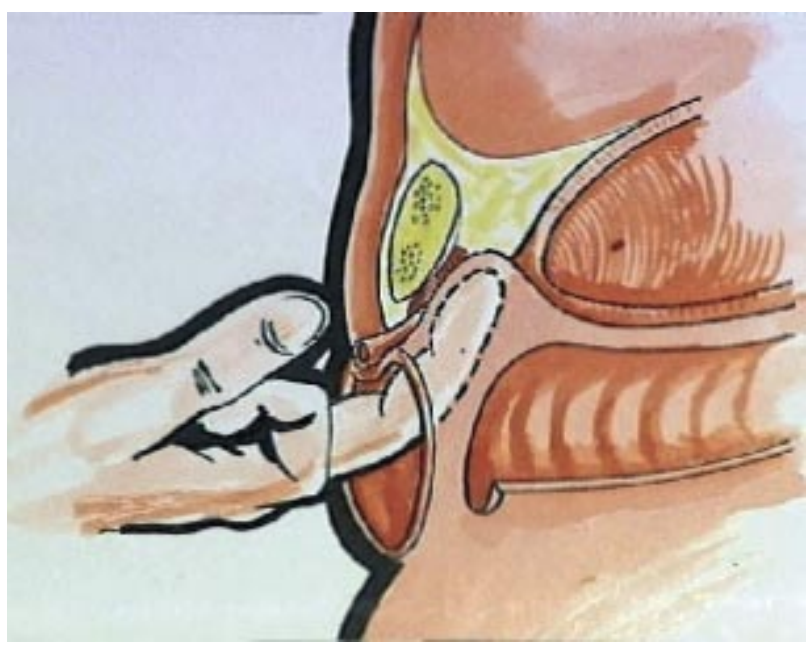

FIGURA 1. Test de V. Bonney unilateral. presenta retención de orina para en poco tiempo después continuar con el escape en la misma o mayor intensidad que antes de la intervención. Sentí rubor al no saber dar explicación a aquel fracaso y la razón no fue otra que mi desconocimiento en la etiopatogenia de lo que estaba tratando. Me empezó a inquietar qué provocaba la incontinencia de orina al esfuerzo, y con la observación y con el Test de V. Bonney descrito en 1923 (4), fuí tomando consciencia de que la continencia se aseguraba fijando la uretra por medio de la vagina a una estructura inmóvil. Así concebí el Test de Bonney unilateral pues sujetando la uretra (por medio de la vagina) contra el pubis en un solo lado se consigue el mismo beneficio. Ahora empezaba la búsqueda de como fijarla mecánicamente pues tenía claro que por proximidad, un punto de anclaje podría ser el propio hueso del pubis. Busqué la bibliografía necesaria y cada vez me afirmaba en que todas las técnicas descritas tenían como denominador común "el anclaje de la uretra".

\section{MATERIAL Y MÉTODO}

Dediqué atención especial a las pacientes con incontinencia de esfuerzo y establecí una consulta monográfica para esta disfunción. Por entonces esta patología no era muy atractiva para los pioneros de la incontinencia (ginecólogos), tal vez, porque los tratamientos que se ofrecían no eran muy eficaces y me atrevería a decir que evitaban el compromiso (al menos, así recibía el mensaje). Todas las pacientes del Área Sanitaria de nuestro Hospital con incontinencia de orina pasaban por mi consulta, tanto las primarias como las ya tratadas, lo que me proporcionó tal bagage que empezaba a encontrarme cómodo con aquella patología.

Con la observación, la exploración física y una profunda búsqueda bibliográfíca aprendí que la ausencia de los surcos uretrovaginales era la consecuencia de la desinserción de la vagina de los ligamentos pubouretrales y con ella la movilidad de la uretra, lo que permite el avance, la exteriorización de estos órganos a la tos provocada: "el aumento de la presión abdominal en cualquiera de sus circunstancias se transmite a la uretra sin pérdida de continuidad provocando fuga del contenido abdominal de no existir los mecanismos de contención". Según la teoría de la continencia que publiqué, es la contracción del diafragma pelviano (cuando participa con su contracción para el aumento de la presión abdominal) y el anclaje de la uretra a través de la vagina (por medio de los ligamentos pubouretrales), los mecanismos que impide el avance de la uretra y la fuga del contenido abdominal (la orina, como contenido de un órgano intraabdominal). 
"Si se pudiera fijar el techo de la vagina contra el pubis, seguro que se consigue la continencia. Se trata de reproducir la maniobra de V. Bonney para mantener permanentemente anclada la uretra" Cada vez que exploraba a una paciente con incontinencia de esfuerzo me ratificaba en mi propósito pero faltaba la forma de hacerlo y que fuera además a través de la vagina (Figura 1), porque la intención era ser lo menos lesivo posible. Con aquel principio empecé a caminar. Comenté la idea con un amigo ingeniero y me remitió a un mecánico de mantenimiento en la fábrica de plástico donde trabajaba, Juan Molino Ortega, al que le explicaba con dibujos y esquemas de libros de anatomía. Era para él impensable realizar un útil que sirviera par una intervención quirúrgica y para ello lo hice venir conmigo al quirófano, para acercarlo lo mas posible a la idea que le quería transmitir. Fuí a visitar en la provincia de Valencia a IQL que fabricaba por entonces prótesis de cadera entre otros elementos sanitarios y desde allí se me orienta al Instituto de Biomecánica en Valencia, (Organismo de la Generalidad Valenciana para el desarrollo tecnológico de procesos mecanizados muy orientado a artilugios ortopédicos).

En cualquiera de los muchos centros y talleres que anduve se me pedía precisión en el objeto a desarrollar, con cotas y dimensiones. Y fué con el primer contacto, con Juan Molino, a quien mejor pude transmitir mi intuición y juntos fuimos dando forma a los artilugios que preparaba. Modificando los diseños nos fuimos acercando a una grapadora accionada con aire comprimido que disparaba las grapas que él confeccionaban artesanalmente con alambre de acero quirúrgico. A las grapas se les remataba

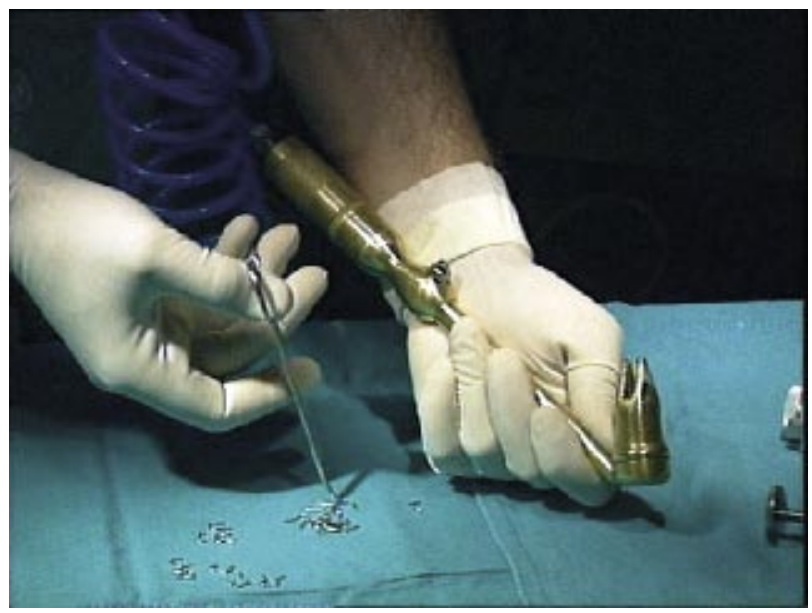

FIGURA 2. Diseño del mecanismo en forma de "pipa", donde se ubican las grapas metálicas para su inserción. los extremos en forma de anzuelo con la intención que fueran autorretentivas. Aunque lo deseable era fijarlas al hueso, la dureza era un reto excesivo a lograr en los primeros momentos por las condiciones de mecanización que se contaba y hubimos de conformarnos en una primera intención con grapar en la sínfisis del pubis. Se hizo la grapadora en forma de "pipa" (Figura 2) para poder atacar el pubis por su cara posterior, pero al tener que ubicarla bajo control radioscópico se hubo de superar la condición metálica acudiendo a otro artesano que confeccionara otra grapadora con material radiotransparente utilizando nylon (Figura 3); la primera grapadora ya dimensionada facilitó el desarrollo de esta otra radiotransparete.

Trabajé en un segundo procedimiento para fijar la vagina al hueso con tornillo autoroscante que lo haría avanzar un destornillador mecánico. Este procedimiento se inició a través de la Cátedra de Ingeniería mecánica de la Universidad de Valencia, pero el tornillo no siempre conseguía penetrar perpendicularmente al hueso y se abandonó el proyecto.

Entre otros compañeros mostré mi idea al Dr. Berián, Jefe del Servicio de la Clínica Universitaria de Pamplona que me ofreció realizar el desarrollo experimental en su Centro, poniendo a mi disposición el animalario, profesionales y toda la infraestructura de que disponía (equipo de radiología, técnica de anestesia, etc.). Esta circunstancia me permitió conocer el manejo del animal y permitirme continuar las sesiones experimentales en mi pueblo con animales comprados en rebaños y con material que me llevara del Hospital.

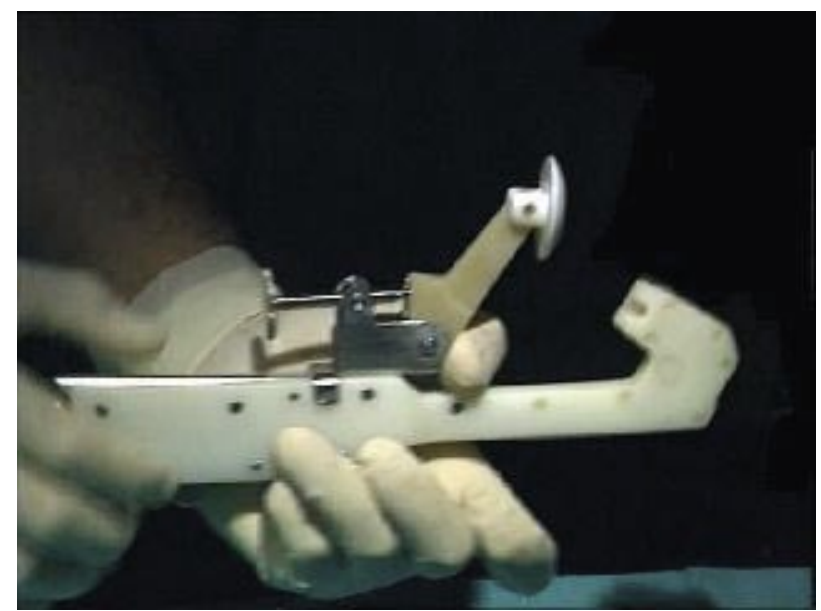

FIGURA 3. Diseño de grapadora radiotransparente con fijación suprapúbica. 
Contacté con la delegación en España de Ethicon y al parecerles interesante el producto fuí invitado a Hamburgo en dos ocasiones, donde estaba instalada la sede para Europa de los procesos de desarrollo de tecnología novedosa. En el primer contacto con la empresa y desde Hamburgo, se realizó una videoconferencia con Cincinaty donde estaba ubicada la Empresa matriz de esta filial de Jhonson and Jhonson. Durante un año estuvimos en relación y me consta que estuvieron trabajando en su diseño pues vino a visitarme un ingeniero desde América y traía consigo un buen fardo de documentos. Pasado este tiempo se me comunica que no era de su interés en ese momento.

También despertó interés a AMS entre otras, que enviaron ingenieros desde América a visitarme. Siempre hube de hacerlo con empresas extranjeras dado que en España no encontré a ninguna en la línea de fabricación.

\section{Técnica Quirúrgica}

La paciente en posición ginecológica; sonda vesical para vacíar la vejiga y la misma sonda servía para delimitar la uretra. Bajo control radioscópico se procuraba que el extremo de la grapadora se apoyara sobre la superficie posterior de la sífisis del pubis. A la grapa se le anudaba un hilo para en caso de no quedar retenida, tirando del mismo se la atraía. Al pulsar el botón disparador, el pistón lanzaba la grapa con suficiente velocidad para permitir clavarse en la sínfisis del pubis (Figura 4). De esta forma, la vagina fijada parauretralmente reconstruía el surco uretrovaginal (Figura 5).

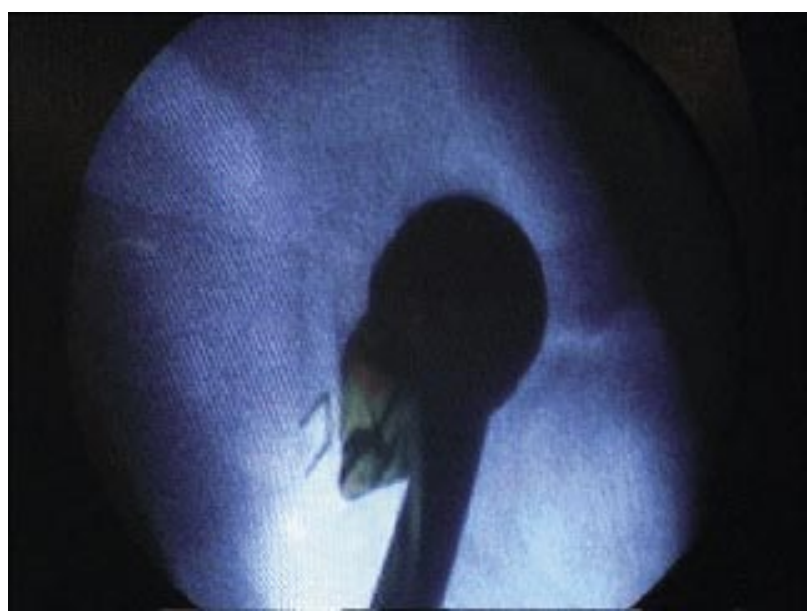

FIGURA 4. Grapa insertada en la sínfisis del pubis.
Traté a 10 pacientes con la técnica y en todas excepto una, se desprendió la grapa antes de que se produjera la cicatrización entre las superficies adheridas. Solo se instalaba una grapa por paciente al tener que limitarme al espacio de la sínfisis.

\section{DISCUSIÓN}

Los trabajos de Delancey de 1989 (5) demuestran que en la porción proximal de la uretra se encuentran los ligamentos que la anclan a través de la vagina al arco tendíneo de la fascia pelviana, lo que le permite la flexión que se produce entre el cuello de la vejiga y la uretra. Es el llamado "ángulo vésico-uretral" posterior que Hodkinson en 1953 (6) y Green en 1962 (7) publican y que posteriormente P. Beck en 1988 (8) lo denomina como efecto"kinking".

Los trabajos de Constantinou y Govan de 1982 (9), y los trabajos de J. Thüroff de 1982 (10) demuestran que en el tercio distal de la uretra se registra una presión que precede y excede a la presión abdominal provocada; y son los trabajos de DeLancey de 1986 (11) los que determinan que la uretra en su recorrido al exterior cruza el músculo diafragmático justo a nivel de su tercio externo, lugar donde se registra la presión en la uretra sana con valores por encima de la presión abdominal.

Estos estudios enseñan que la incontinencia de orina en la mujer es la consecuencia de las lesiones anatómicas que se producen alrededor de la uretra tanto de su anclaje (ligamentos pubouretrales) como en el músculo elevador del ano, con la consecuente pérdida del tono muscular, quedando sin

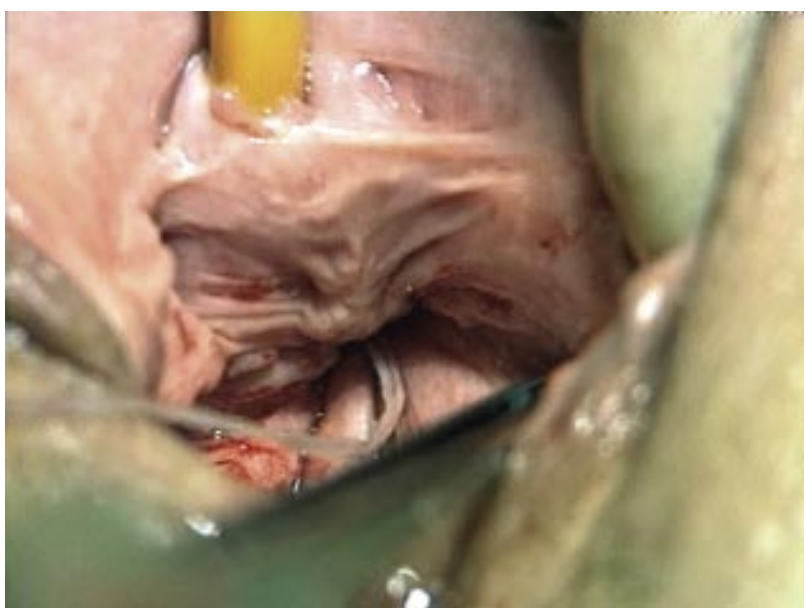

FIGURA 5. Surco uretrovaginal reconstruido. Se observa el detalle del hilo sujeto a la grapa insertada. 
la fuerza necesaria para ocluir la uretra cuando se contrae al participar en la elevación de la presión intrabdominal. Fijando la uretra al pubis (mecanismo de los ligamentos) se detiene la transmisión de la presión al facilitar el pliegue en la uretra proximal. Rehabilitando el suelo pélvico se consigue fortalecer el músculo elevador del ano, aumentando la presión que este músculo ejerce sobre la uretra (cuando se contrae simultaneamente con el resto de los músculos abdominales para elevar la presión intrabdominal).

(En 1998 aparece en escena el procedimiento "In-Fast" de origen israeli, basado en el mismo principio que yo patenté en 1992. El Dr. Slomo Raz lo va mostrando en los Congresos y yo personalmente lo ví utilizar desde el Hotel Firia de Barcelona en retransmisión directa desde el quirófano del Hospital de Badalona).

\section{BIBLIOGRAFÍA y LECTURAS RECOMENDADAS ( ${ }^{*}$ lectura de interés $y^{* *}$ lectura fundamental)}

1. $\mathrm{N}^{\circ}$ de Patente ES 2049645.

2. RAZ, S.: "Modified bladder neck supension for female stress incontinence". Urology, 17: 82, 1981.
3. PEREYRA, A.J.: "Simplified surgical procedure for the correction stress incontinence in women". Surg. Obstet. and Gynaecol., 1959.

4. BONNEY, V.: "On diurnal incontinence of urine in woman". Journal of Obstetrics and Gynaecology, 358-365, 1923.

5. DeLANCEY, J.O.L.: "Pubovesical ligament: a Separate Structure from the Uretrhal Supports ("Pubouretrhal ligaments")". Neurourology and Urodinamics, 8: 53, 1989.

6. HODGKINSON, C.P.: "Relationships of the female urethra and bladder in urinary stress incontinence". Am. J. Obst. and Gynec., 65: 560, 1953.

7. GREEN, T.H.: "Development of a plan for the diagnosis and treatment of urinary stress incontinence". Am. J. Obst. and Gynec., 83: 632, 1962.

8. BECK, P.: "Intraurethral-intravesical cough-presure spikedifferences in 267 patientes surgically cured of genuine stress incontinence of urine". Obstet. and Gynecol., 73: 302, 1988.

9. CONSTANTINOU, C.E.; GOVAN, D.E.: "Spatial distribution and timing of transmitted and reflexly generated urethral pressures in healthy women". J. Urol., 127: 964, 1982.

10. THÜROFF, J.: "Mechanisme of urinary continence : an animal model to study urethral response to stress conditions". J. Urol., 127: 1202, 1982.

11. DeLANCEY, J.O.L.: "Correlative Study of Paraurethral Anatomy". Obstetrics and Gynaecology, 68: 91, 1986. 